


\title{
La auditoría de valores razonables: riesgos y desafíos para el auditor
}

\author{
Audit of fair values: risks and challenges for the auditor \\ Eduardo Sosa Mora* \\ Recibido: 21/6/2016 / Aprobado:1/11/2016 \\ Resumen
}

\begin{abstract}
En las Normas Internacionales de Información Financiera (NIIF) se ha venido transitando, desde hace tres décadas, del costo histórico hacia el valor razonable como principal modelo de medición contable, lo cual constituye un cambio paradigmático que permite representar las partidas de los estados financieros de acuerdo con los valores de mercado.. En este artículo se exponen los principales riesgos y desafíos que enfrentan los auditores al examinar partidas de los estados financieros preparados sobre la base del valor razonable, lo mismo que las nuevas oportunidades para su desarrollo profesional que esto trae aparejado.
\end{abstract}

Palabras clave: valor razonable, medición contable, auditor, auditoría, estados financieros, riesgos de incorrección material.

\begin{abstract}
International Financial Reporting Standards (IFRS) has been gradually passing, during the last three decades, from the historical cost to fair value as the main model of accounting measurement, which is a paradigm shift that allows representing the elements of the financial statements according to their market values. This article describes the main risks and challenges facing auditors to examine the elements of financial statements prepared according with fair value, as well as new opportunities for professional development that this brings.
\end{abstract}

Key Words: fair value, accounting measurement, auditor, audit, financial statements, material risks inaccuracy

\section{Introducción}

El ascenso del valor razonable como principal modelo de medición en las NIIF constituye un cambio paradigmático en la disciplina contable, que contribuye a satisfacer las complejas necesidades de información de los usuarios de los estados financieros en el mundo globalizado, pero también trae consigo importantes riesgos y desafíos para los auditores que dictaminan esos estados.

La creciente dependencia de las cifras contables respecto de variables externas a las empresas, así como de hipótesis sobre el comportamiento de los mercados y estimaciones sobre eventos futuros inciertos, se traduce en nuevos y más complejos riesgos de incorrección material en los estados financieros, debido principalmente a la influencia del juicio de la dirección en la selección y aplicación de las metodologías, modelos y técnicas para calcular valores razonables de activos y pasivos, todo lo cual conduce a que la responsabilidad social del auditor (proveer confianza a los usuarios en los estados financieros) adopte nuevas dimensiones.

Se impone la obligación de realizar cambios sustanciales en el enfoque de la auditoría de estados financieros, lo mismo que en los procedimientos y las técnicas para realizarla, y transitar desde una óptica esencialmente corroborativa de la exactitud

\footnotetext{
* Magister en Evaluación de Programas y Proyectos de Desarrollo y Licenciado en Administración de Negocios con énfasis en Contabilidad, Escuela de Administración de Negocios. Universidad de Costa Rica. Correo electrónico: eduardo.sosamora@ ucr.ac.cr
} 
de las cifras contables hacia un enfoque más crítico y evaluativo acerca de la validez, la relevancia y la credibilidad de esas cifras.

Esos riesgos y desafíos vienen acompañados de nuevas oportunidades para el desarrollo profesional porque los auditores están ahora en la obligación de expandir las fronteras de sus conocimientos y habilidades más allá de los límites de la contabilidad y la auditoría, para incursionar en temáticas como la valoración de activos y de empresas, la modelación financiera, los métodos estadísticos y el funcionamiento de los diferentes mercados, entre otras; lo que les facilitará interactuar con diferentes especialistas de los equipos interdisciplinarios de auditoría en el ejercicio de su responsabilidad de dictaminar sobre la razonabilidad de los estados financieros.

\section{La auditoría de estados financieros: naturaleza y objetivos}

De acuerdo con la Norma Internacional de Auditoría 200 (NIA 200) el objetivo de una auditoría es aumentar el grado de confianza de los usuarios en los estados financieros, por medio de la expresión de una opinión del auditor respecto a si dichos estados han sido preparados, en todos los aspectos significativos, en conformidad con las normas de información financiera establecidas por el organismo emisor autorizado o reconocido, o por requerimientos legales o reglamentarios que la entidad haya adoptado como marco de información financiera (IFAC, 2011).

La responsabilidad fundamental del auditor consiste en obtener evidencia suficiente y adecuada para alcanzar un grado razonable de seguridad y formarse una opinión respecto a si los estados financieros sometidos a su examen están exentos de representaciones erróneas significativas, debidas ya sea al fraude o al error y, por lo tanto, presentan razonablemente la situación financiera, el rendimiento financiero, los cambios en el patrimonio neto y los flujos de efectivo de la entidad, en conformidad con las Normas Internacionales de Información Financiera (NIIF), cuando sea este el marco de información financiera aplicable a la entidad.
Para lograr ese objetivo, el auditor -entre otras responsabilidades- identifica y valora los riesgos de que existan incorrecciones materiales en los estados financieros, mediante el conocimiento de la entidad, su entorno y su control interno. Asimismo, obtiene evidencia adecuada y suficiente por medio del diseño y la ejecución de procedimientos apropiados en respuesta a los riesgos evaluados (IFAC, 2011); se incluye en el proceso la planificación y ejecución de tareas sustantivas para comprobar si las representaciones de los estados financieros se ajustan a los requerimientos establecidos en las NIIF, en los cuales se establecen los lineamientos que debe seguir la entidad en relación con el reconocimiento, la medición, la presentación y la revelación de las partidas de los estados financieros.

En lo que respecta específicamente a los requerimientos relativos a la medición de los importes por los cuales se expresan esas partidas (medición contable), el auditor ejecuta procedimientos sustantivos para comprobar que los métodos y las técnicas utilizados por la entidad para obtener esos importes se ajusten a las normas de información financiera específicas relativas a cada una de las partidas de los estados financieros.

\section{La medición contable}

Acorde con el marco conceptual para la Información Financiera (IASB, 2010), las NIIF permiten el empleo de diferentes modelos de medición contable, a saber, el costo, el valor razonable, el valor en uso, el valor revaluado (que se basa en el valor razonable) y el valor neto realizable.

Durante muchos años, el costo histórico fue el principal modelo de medición contable (Gómez y Álvarez, 2013). Sin embargo, a raíz de la globalización económica y del mayor desarrollo de los mercados financieros, dicho modelo comenzó a perder relevancia por su poca representatividad de la realidad de los negocios, principalmente porque basa las mediciones contables en los importes acordados en transacciones realizadas en el pasado, información que pierde vigencia frente a la dinámica de los mercados en el mundo globalizado (González, Martínez y Requiterena, 2010). 
El modelo del valor razonable comenzó a adquirir mayor auge en las NIIF a partir de la década de los años mil novecientos noventa (Silva y Azúa, 2006; Suárez y Lorca, 2007; Gómez, et al, 2011; Gómez y Álvarez, 2013 y Jeppesen y Liempd, 2015), este propone la medición de los activos y pasivos según sus valores de mercado. Como aportan Montilla y Cante (2004): "Un hecho que se puede constatar fácilmente es que las mediciones y revelaciones hechas a valor razonable, de manera creciente, se están volviendo poderosamente dominantes en las estructuras conceptuales del proceso de presentación de reportes financieros" (Montilla y Cante, 2004:71)

Su aceptación ha crecido en forma vertiginosa en las últimas tres décadas y se ha ido incorporando paulatinamente en las NIIF hasta culminar, en el año 2011, con la emisión de la Norma Internacional de Información Financiera 13 (NIIF 13) Medición del valor razonable (IASB, 2011). En el anexo 1 se exponen las partidas cuya medición mediante el valor razonable es optativa u obligatoria, tanto en el reconocimiento inicial como en las mediciones posteriores según las NIIF.

\section{La medición contable según el valor razonable}

Según la NIIF 13 (IASB, 2011), el valor razonable es el precio que se recibiría por la venta de un activo o que se pagaría por la transferencia de un pasivo en una transacción ordenada en el mercado principal (o en el mercado más ventajoso) en la fecha de la medición, en condiciones de mercado presentes. Se trata de un precio de salida que puede ser observable de manera directa, o bien, puede estimarse por medio de otra técnica de valoración.

Una medición del valor razonable se refiere a un activo o un pasivo concreto. Por ello, al efectuar la medición, se deberán tener en cuenta las características del activo o pasivo, de la misma manera en que los participantes de mercado las tendrían al acordar el precio en la fecha de la medición. La NIIF 13 (IASB, 2011) dispone que la medición del valor razonable debe realizarse a partir de los supuestos que los participantes de mercado utilizarían para fijar el precio, al asumir que estos actúan en su mejor interés económico.
Esa norma distingue entre variables y técnicas. Las primeras son las hipótesis que los participantes de mercado emplearían al determinar los precios para los activos o pasivos; las segundas consisten en los métodos y procedimientos específicos para calcular los valores razonables.

Las variables se dividen en observables y no observables. Las observables se obtienen a partir de datos de mercado, como la información pública sobre los sucesos o transacciones reales. Las no observables son aquellas para las cuales no se cuenta con datos de mercado y se formulan a partir de la mejor información disponible sobre los supuestos en que los participantes de mercado se apoyarían al establecer los precios.

Por otra parte, mediante las técnicas de valoración se determina el valor razonable. La NIIF 13 (IASB, 2011) menciona tres enfoques de valoración: el enfoque de mercado, el enfoque del costo y el enfoque del ingreso.

El enfoque de mercado utiliza los precios y otra información relevante generada por transacciones de mercado que involucran los activos y pasivos objeto de medición, o un grupo de activos y pasivos idénticos o comparables. El enfoque del costo muestra el monto que se requeriría para sustituir la capacidad de servicio de un activo. Por último, el enfoque del ingreso convierte importes futuros de efectivo en un importe presente único descontado. La medición del valor razonable, según este enfoque, se determina sobre la base del valor indicado por las expectativas presentes de mercado sobre esos importes futuros. Todos estos elementos se representan en la Figura 1. 
Figura 1

\section{Variables y técnicas para la medición contable con base en el valor razonable}

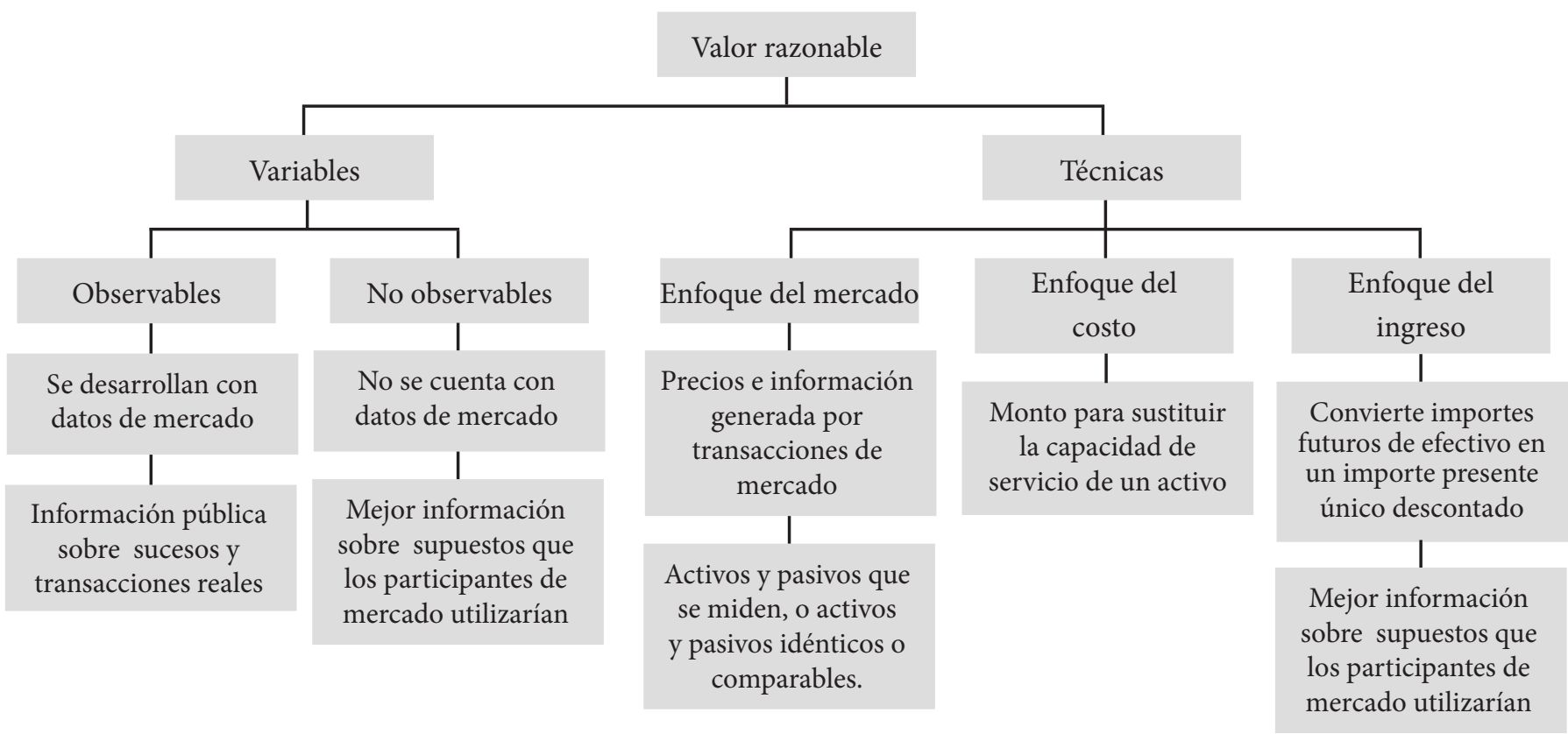

Acorde con la NIIF 13 se pueden utilizar las técnicas de valoración apropiadas en las circunstancias, y sobre las cuales se disponga de datos suficientes para medir el valor razonable, además, se debe maximizar el uso de variables observables y minimizar el uso de variables no observables. Esta norma establece una jerarquía que ordena en tres niveles las variables que se emplean para determinar dicho valor, en la cual se otorga la prioridad más alta a los precios cotizados en mercados activos para activos y pasivos idénticos (Nivel 1), y la más baja a las variables no observables (Nivel 3). Esto se resume en la Figura 2:

\section{Figura 2}

\section{Jerarquía (niveles) para determinar el valor razonable según NIIF 13}

Nivel 1 Precios cotizados en mercados activos para activos o pasivos idénticos

\begin{tabular}{|l|l|} 
Nivel 2 & $\begin{array}{l}\text { Precios cotizados para activos o pasivos similares en mercado activos. } \\
\text { Precios cotizados para activos o pasivos idénticos o similares en mercados no } \\
\text { activos }\end{array}$ \\
\hline Nivel 3 & Son variables no observables. \\
& Se utilizan cuando no estén disponibles variables observables relevantes \\
\hline
\end{tabular}


Las variables del nivel 1 proporcionan la evidencia más fiable del valor razonable y, por esto, cuando estén disponibles, se deben utilizar sin ajuste para medir dicho valor, para lo cual es necesario determinar el mercado principal para el activo o pasivo, o en su defecto, el mercado más ventajoso. Con respecto a las variables del nivel 3 señala la NIIF 13 que estas deben incorporar los supuestos que los participantes de mercado utilizarían al acordar los precios, incluyendo los referidos al riesgo.

\section{Ausencia de datos observables: el enfoque del} ingreso y variables del nivel 3

Aunque la NIIF 13 privilegia el empleo de las variables observables, en ausencia de mercados activos puede imposibilitarse disponer de información de variables de los niveles 1 y 2 para tal propósito, en cuyo caso, el cálculo del valor razonable debe realizarse a partir de variables no observables (nivel 3) y se debe aplicar el enfoque del ingreso, según el cual, el valor razonable equivale al valor presente de los importes futuros esperados de un activo o pasivo. Cuando se emplea esta técnica, según la NIIF 13, la medición del valor razonable representa las expectativas del mercado sobre los importes futuros esperados, de manera que este valor es el resultado de estimaciones y expectativas sobre eventos futuros.

La aplicación de técnicas de valor presente para descontar cifras obtenidas con base en estimaciones sobre sucesos futuros para medir el valor razonable, es un hecho aceptado por la NIIF 13 (IASB, 2011), donde se expone que el uso de esas técnicas se realiza bajo condiciones de incertidumbre en cuanto a los montos y a la distribución temporal de esos flujos.

El cálculo del valor razonable según el enfoque del ingreso incluye los elementos que se exponen en la Figura 3.

Figura 3

\section{Elementos (variables) para el cálculo del valor razonable de activos y pasivos Según el enfoque del ingreso}
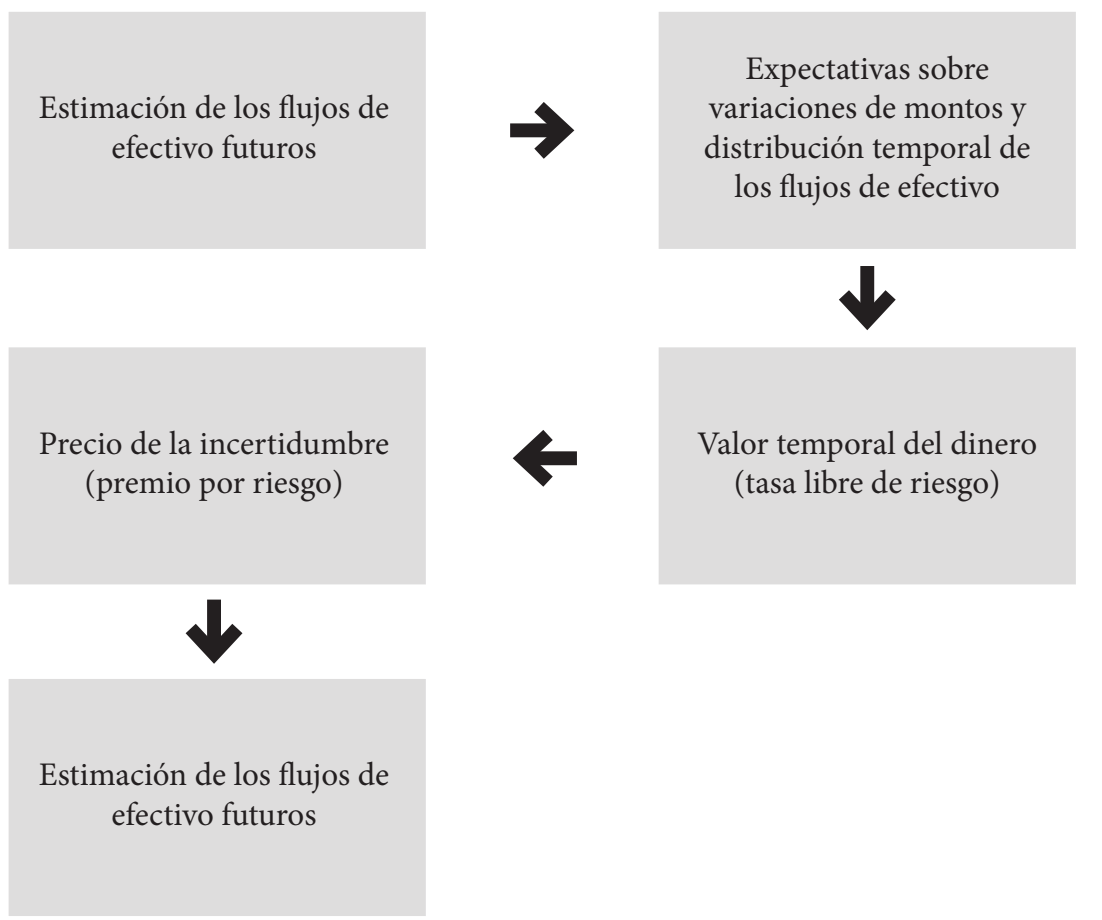
Según la NIIF 13 (IASB, 2011) las técnicas de valor presente empleadas en la aplicación del enfoque del ingreso deben "captar" esos elementos desde la perspectiva de los participantes en el mercado. Para efectuar estas estimaciones, dicha norma establece los principios generales que se exponen en la Figura 4.

$\mathrm{Al}$ seguir este enfoque, los valores razonables se obtienen al despejar la fórmula del valor actual de una serie de importes futuros:

\section{VALOR RAZONABLE $=\sum_{t=1}^{n} \frac{F N E t}{(1+k)} t$}

En donde con FNE se representan los flujos netos de efectivo anuales esperados de los activos o pasivos de cada periodo, $\mathbf{n}$ representa el número de años durante los cuales se espera recibir o pagar esos flujos, $\mathbf{t}$ representa cada uno de los $\mathbf{n}$ años en que el activo o pasivo generan flujos de efectivo y $\mathbf{k}$ es la tasa de rendimiento para descontar los flujos (FNE)

La tasa de descuento $\mathbf{k}$ está determinada por la compensación por la pérdida de del valor del dinero en el tiempo (tasa libre de riesgo), el premio por asumir el nivel de riesgo del activo o pasivo y otros factores que consideren los participantes del mercado (como podría ser una compensación adicional al inversionista) por asumir el riesgo país.

En vista de la incertidumbre que rodea a las estimaciones de eventos futuros, la NIIF 13 (IASB, 2011) permite que los flujos de efectivo anuales sean valores promedios esperados de los flujos netos asociados a los distintos escenarios proyectados, multiplicados por las respectivas probabilidades asignadas:
Donde FNE i es el flujo de efectivo neto asociado al escenario i, Pi la respectiva probabilidad de ocurrencia y E (FNE t) es el valor esperado de los flujos netos de efectivo del año $t$.

Las mediciones de los valores razonables en sucesivos periodos generarán variaciones con respecto a los importes registrados, las cuales se reconocerán en el estado de resultados integral en algunos casos (instrumentos financieros al valor razonable con cambios en resultados, propiedades para inversión y activos biológicos), o bien, como parte de otro resultado integral (propiedad, planta y equipo y activos intangibles).

Para medir el valor razonable se sigue el enfoque del ingreso, la NIIF 13 propone dos técnicas: la del ajuste a la tasa de descuento y la del valor presente esperado. Para esta última, a su vez, proponen dos métodos, identificados como 1 y 2 . Estas técnicas difieren en el tipo de flujo de efectivo que utilizan y en los métodos de ajuste por el riesgo que aplican, como se expone en el Anexo 2.

FNE t $=\mathrm{E}($ FNE t $)=\sum_{i=1}^{n} F N E i x \boldsymbol{P i}$ 


\section{Figura 4}

\section{Principios para aplicar la técnica del valor presente para determinar valores razonables}

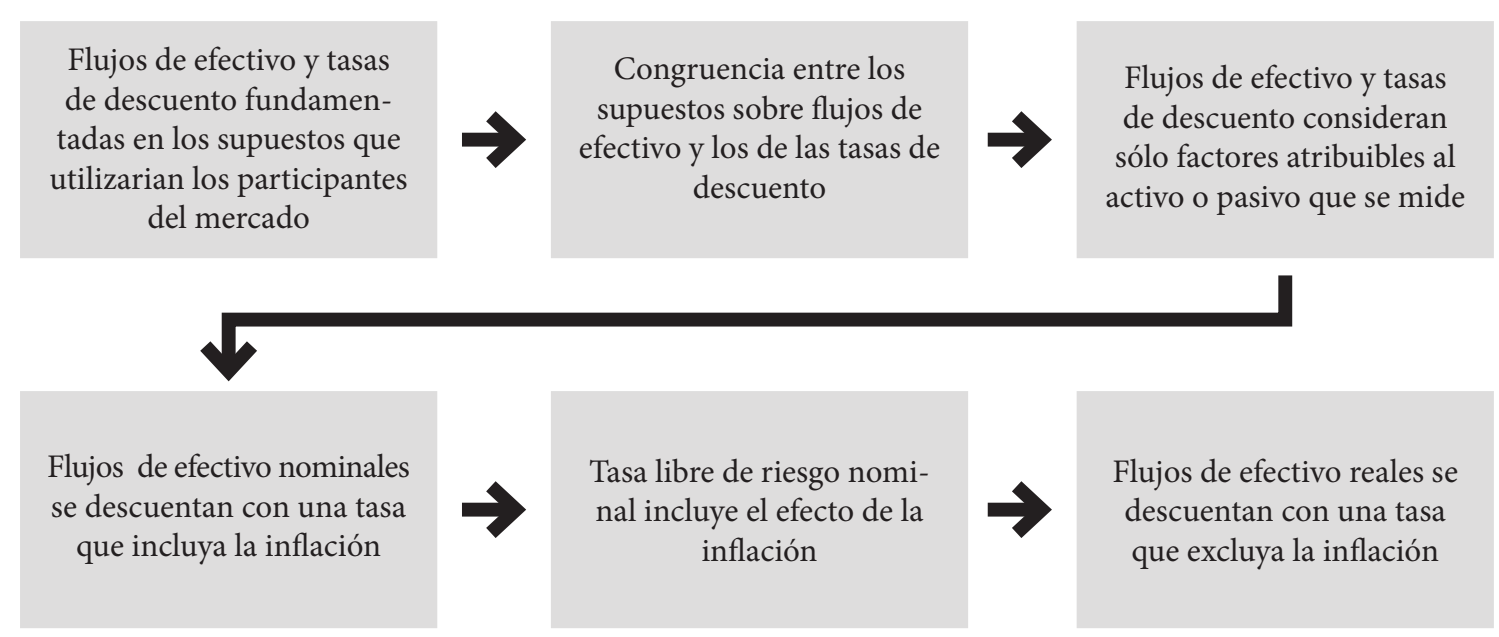

\section{Del costo histórico al valor razonable: los nuevos riesgos de medición}

El advenimiento del valor razonable como principal modelo de medición constituye un cambio paradigmático en la contabilidad (Valle, 2009; Gómez, De la Hoz y López, 2011; Silva, 2011 y Jeppesen y Liempd, 2015), que si bien, facilita la satisfacción de las necesidades de los usuarios de los estados financieros, también genera importantes riesgos. Como señalan Morala, Fernández, Fernández y Gutiérrez (2010), la aplicación del valor razonable conlleva una serie de retos: el propio objetivo de la medición; la disponibilidad y fiabilidad de la evidencia; los activos y pasivos a los que debe aplicarse; la diversidad y complejidad de métodos de valoración, así como a formulación de juicios sobre hipótesis significativas derivados de la opinión de los expertos.

Del estático costo histórico, que anclaba al pasado los importes de las partidas de los estados financieros, la Contabilidad ha evolucionado hacia la representación de las cifras de acuerdo con datos de mercado, tales como los precios cotizados para activos y pasivos idénticos o similares, o bien, de acuerdo con las expectativas y supuestos de los participantes en el mercado acerca de los flujos de efectivo esperados de activos y pasivos. El costo histórico informa acerca del monto desembolsado cuando se adquirió un activo, el valor razonable dice cuál es su precio de salida; es decir, su valor de mercado.

Uno de los riesgos asociados con este cambio es el efecto de la volatilidad de los mercados sobre los estados financieros, que podría redundar en el reconocimiento de ganancias por variaciones efímeras de los valores de mercado de activos y pasivos y podría crear la ilusión efímera de incrementos del valor de los activos y del rendimiento financiero.

Otro riesgo reside en la dependencia de las cifras contables respecto de la formulación de supuestos y estimaciones sobre eventos futuros, sujetos a la influencia del juicio o del criterio de la dirección. Con el fin de medir el valor razonable, la dirección prepara la información relevante en la fecha de cada medición, en la cual se incluye un conjunto de factores causales para su determinación, así como una ponderación de estos factores, lo que 
inevitablemente aumenta el riesgo de error en los estados financieros (Jeppesen y Liempd, 2015).

De acuerdo con Gómez (2009) la responsabilidad del auditor al examinar los estados financieros es mayor porque al presentarse saldos basados en estimaciones de valores razonables, el riesgo de que existan errores importantes aumenta significativamente, sobre todo si se tiene presente que tales estimaciones pueden ser influenciadas por la administración de la entidad. Puede existir sesgo de la dirección en la selección de las hipótesis y las técnicas para calcular valores razonables que resulten en representaciones distorsionadas de los estados financieros.

\section{Riesgos y desafíos de la auditoría de valores razonables}

Surge, ante el panorama actual, la interrogante sobre cómo se puede garantizar a los usuarios de los estados financieros la razonabilidad de las hipótesis de la dirección sobre cantidades, precios, tasas de inflación, ritmo de crecimiento de las ventas y tasas de descuento, entre otras variables; así como quién garantiza que las premisas que subyacen a las cifras obtenidas representan las expectativas y supuestos de los participantes del mercado, como lo requiere la NIIF 13.

Aun en el caso de que la dirección de la entidad se apoye en especialistas para obtener los valores razonables, ello no la libera de sus responsabilidades en relación con la preparación de los estados financieros, ni tampoco a los auditores con respecto a la opinión que emiten. Como apunta Gómez (2009):

La precaución de que dichas estimaciones sean realizadas por un tasador externo, a mi juicio, no liberan al auditor de revisar adecuadamente las bases utilizadas para estimar el valor razonable de los recursos (obligaciones) de una entidad, así como tampoco liberan a la entidad a desarrollar sus propios modelos de valuación... (Gómez. 2009:43)
Un riesgo adicional se relaciona con el grado de conocimiento de los profesionales contables sobre la naturaleza, funcionamiento y características de los diferentes mercados de donde se deben obtener los datos para calcular los valores razonables, ante lo que surge la inquietud de si estos profesionales realmente están en capacidad de evaluar si la información de entrada para ese propósito es adecuada y razonable para preparar los estados financieros.

Las transformaciones en el paradigma de la contabilidad obligan a realizar cambios sustanciales en el enfoque de la auditoría de estados financieros, lo mismo que en los procedimientos y las técnicas para realizarla. Según Montilla (2008), la consolidación de la contabilidad a valor razonable ejerce presión para que se audite a valor razonable.

Los estados financieros son a la vez el producto final de la contabilidad y el objeto de estudio de la auditoría, si cambia la contabilidad también debe cambiar la auditoría; sin embargo, según Jeppesen y Liempd (2015), el cambio en el paradigma contable aún no ha ejercido los efectos esperados en el paradigma principal de la auditoría porque afirma que:

los cambios ontológicos, epistemológicos y metodológicos en la visión de mundo de la contabilidad, tienen una influencia ontológica, epistemológica y metodológica en la visión de mundo de la auditoría. Si una cambia, la otra debe cambiar también, con el fin de mantener una relación válida entre contabilidad (objeto de estudio) y auditoría. (Jeppesen y Liempd, 2015:39)

Los procedimientos y las técnicas de auditoría para comprobar la razonabilidad de los importes históricos de los activos y pasivos pueden no ser efectivos para auditar valores razonables, incluso algunas técnicas y procedimientos podrían ser totalmente irrelevantes para tal fin.

La auditoría de los importes obtenidos con base en el costo histórico se apoya, principalmente, en el empleo de técnicas como el examen de documentos y la inspección física, pero para auditar los valores razonables eso no es suficiente. 
Por ser el valor razonable una estimación contable, el auditor debe planificar y ejecutar metodologías que le permitan ir más allá de la simple constatación de saldos. Es insuficiente verificar que los valores razonables sean consistentes con las metodologías y los modelos seleccionados por la dirección, más que eso, el auditor debe examinar los fundamentos mismos, la relevancia y la adecuación de esas metodologías y modelos.

Después de obtener entendimiento acerca de los métodos y modelos con base en los cuales la dirección mide los valores razonables, los auditores deben comprobar que las cifras de los estados financieros hayan sido obtenidas conforme con las metodologías establecidas por la dirección, es decir, comprobar si esas metodologías son aplicadas de manera apropiada y coherente, según lo requiere la NIA 540 (IFAC, 2011).

Adicionalmente, los auditores deben evaluar la razonabilidad de las variables y los supuestos en que se apoya esa metodología, tales como los precios de bienes idénticos; los precios de bienes similares negociados en mercados activos o en mercados no activos; los modelos y técnicas financieras utilizados (enfoque del ingreso); las tasas de rentabilidad mínima esperada para descontar los flujos (tasas libres de riesgo, indicadores de riesgo sistemático, primas de riesgo), lo mismo que la viabilidad de las premisas sobre los flujos futuros de efectivo del activo o pasivo objeto de medición. Deben los auditores, además, analizar si en esas cifras se recogen apropiadamente las perspectivas de los participantes en el mercado, como lo requiere la NIIF 13.

El auditor debe analizar porqué la dirección selecciona y utiliza determinadas variables del mercado y no otras para medir los valores razonables, es decir, debe evaluar los criterios de selección de los datos relevantes para las mediciones contables. También puede aplicar otros modelos alternativos para calcular los valores razonables y analizar las diferencias entre las cifras obtenidas al aplicar estos modelos y las obtenidas por la dirección, o bien, plantear sus propias hipótesis con el fin de evaluar la sensibilidad de las mediciones establecidas por la dirección ante cambios en las variables del modelo y conocer el grado de riesgo de las estimaciones efectuadas por la dirección.

La auditoría de los valores razonables no puede ser delegada completamente en los expertos. Si bien, el auditor puede contar con el apoyo de expertos, él es el único responsable de la opinión sobre los estados financieros. Por esto, el auditor no debe limitarse a incorporar las valoraciones realizadas por los expertos y tomarlas como evidencia válida ni "puede convertirse en un administrador de subcontratos cuyos resultados incorpora como evidencia de auditoría" (Montilla, 2008:34).

A manera de ilustración, se pueden comparar los procedimientos de auditoría que se aplicarían para verificar los saldos de las Propiedades para Inversión, cuando estas se miden según el costo histórico y cuando se miden de acuerdo con el valor razonable, ambos aceptados por la Norma Internacional de Contabilidad 40 (IASB, 2011) para la medición posterior de estas propiedades.

Para comprobar la corrección del costo de las propiedades, el auditor examina los documentos de respaldo respectivos, como cheques, transferencias de fondos, facturas o recibos de dinero, pagarés o hipotecas y también comprueba la correcta valuación de estas propiedades, en lo que se refiere a la inclusión de todos los desembolsos capitalizables como honorarios profesionales de notarios, arquitectos e ingenieros, impuestos por el traspaso de las propiedades, entre otros, lo mismo que la autenticidad de esos documentos. Es decir, aplica procedimientos sustantivos esencialmente corroborativos.

En contraste, para verificar los valores razonables de esas propiedades, en caso de existir información de mercado (variables observables), el auditor debe identificar los mercados principales o los más ventajosos para obtener los datos relativos a los precios de salida y comprobar si los precios utilizados por la dirección son consistentes con los de esos mercados; asimismo, debe confirmar esos datos con las respectivas fuentes de información, así como evaluar la relevancia y la confiabilidad de estas. 
Si la entidad utiliza el enfoque del ingreso, el auditor debe evaluar la viabilidad de las hipótesis subyacentes a esas mediciones en relación con el comportamiento del mercado. Esto incluiría analizar, entre otros aspectos, si las proyecciones de ingresos por arrendamientos se basan en precios y niveles de ocupación realistas en las condiciones actuales del mercado inmobiliario específico para esas propiedades; si las tasas de crecimiento previsto de la ocupación y de los precios de los alquileres en cada zona son realistas; si los modelos matemáticos empleados para proyectar los niveles de ocupación y las fuentes de información de las que estos se alimentan son apropiados en las circunstancias y si los valores residuales previstos son adecuados tomando en cuenta factores como la plusvalía inmobiliaria de la zona.

Además, el auditor tiene que evaluar si las tasas de descuento de los flujos de efectivo son representativas de la rentabilidad esperada y el riesgo de las inversiones en el mercado inmobiliario, así como también sensibilizar los resultados de las proyecciones ante cambios en las variables críticas, e incluso, formular sus propias estimaciones empleando el mismo modelo de la dirección u otros modelos alternativos.

En el anexo 3 se presenta una lista sugerida de objetivos y procedimientos básicos para auditar valores razonables de diferentes partidas. Esta es una lista de procedimientos generales y básicos que se sugieren para tal fin. No se pretende que esa lista sea exhaustiva y para su ejecución el auditor puede necesitar del aporte de expertos en diferentes materias.

Se pueden identificar cuatro desafíos que el auditor enfrenta con la consolidación de la contabilidad basada en valores razonables (Tejada, 2012). El primero es la necesidad de obtener mayores conocimientos en otras áreas (finanzas, economía, estadística y funcionamiento de los diferentes mercados, entre otras. En estrecha relación, el segundo consiste en la necesidad de capacitarse de manera permanente en esas temáticas. El tercero es la creciente necesidad de auditor de utilizar trabajos y opiniones de expertos en diferentes campos, sin que esto lo libere de su responsabilidad de dictaminar los estados financieros y el último reside en la necesidad de desarrollar habilidades para lidiar con estimaciones y evidencias subjetivas de parte de la dirección, la que podría estar sesgada hacia la aplicación de solamente ciertos métodos o técnicas.

\section{Síntesis y conclusiones}

El nuevo paradigma de la medición contable basada en el valor razonable plantea importantes desafíos a la auditoría de estados financieros. La determinación de los valores razonables de activos y pasivos está sujeta a la influencia múltiples factores de índole subjetiva lo cual incrementa el riesgo de que existan incorrecciones materiales en los estados financieros y, consecuentemente, aumenta el riesgo que enfrenta el auditor de opinar que los estados financieros están exentos de representaciones erróneas significativas, conforme con las NIIF, cuando en realidad sí están distorsionados por errores importantes.

El auditor de las nuevas generaciones no podrá ser un profesional cuyos conocimientos se circunscriban a la contabilidad y a la auditoría. Todo lo contrario, para estar en condiciones de garantizar a los usuarios de los estados financieros que estos se ajustan -en todos los aspectos significativos- a las NIIF, el auditor tendrá que cuestionar con escepticismo profesional y (más allá de constatar o verificar datos y de reprocesar cálculos) evaluar los cimientos conceptuales y la razonabilidad de las hipótesis de las metodologías utilizadas por las dirección para estimar los valores razonables. Deberá emplear modelos y métodos alternativos a los de la entidad que audita y realizar análisis de sensibilidad como parte de su trabajo de identificación y valoración de riesgos de incorrección material.

Estos riesgos y desafíos se pueden transformar en oportunidades para el desarrollo profesional de los auditores, quienes estarán en la obligación de adquirir y mantener actualizados conocimientos en temas como valoración de activos, de empresas, de acciones, bonos y otros instrumentos financieros más complejos; funcionamiento de los mercados 
financieros, inmobiliarios y de activos biológicos, entre otros; modelación financiera probabilística, simulación y métodos estadísticos de pronóstico, todo ello para cumplir su función social de mantener la confianza de los usuarios en los estados financieros que emiten las empresas.

Se deberá superar la creencia de que la determinación de valores razonables es asunto exclusivo de expertos y que los auditores se limitan a aportar como evidencia los informes de los especialistas. Aunque tanto la dirección de las empresas como los auditores continuarán apoyándose en los aportes de expertos en diferentes materias (más bien, es probable que esta necesidad del auditor crezca), la responsabilidad social que asume el auditor frente a todos los usuarios y los eventuales perjuicios económicos que puede causar a la sociedad la circulación de estados financieros distorsionados imponen a este profesional el imperativo moral de adquirir los conocimientos, habilidades y experiencia necesarias para lidiar con mayores grados de complejidad en su trabajo, con especialistas y con el riesgo de estimaciones sesgadas por parte de las empresas que audita.

De manera concomitante con los cambios en el espectro de conocimientos y habilidades que se esperan de los auditores para encarar las exigencias de los usuarios de la información financiera en el mundo globalizado, los auditores deberán transitar del enfoque corroborativo de la exactitud de las cifras contables (costo histórico) hacia un enfoque más evaluativo de la validez, viabilidad y credibilidad de esas cifras (valor razonable). El cambio del paradigma de medición en la contabilidad demanda cambios en los enfoques de la auditoría.

La enseñanza para la formación de profesionales de la contaduría también deberá cambiar. Temas como la evaluación de proyectos, la valoración de empresas, los pronósticos y la modelación financiera y estadística, los mercados financieros, la contabilidad y la auditoría financiera, entre otros, no deberán seguir siendo abordados de forma aislada e inconexa, sino que deberán ser estudiados en cursos integrados impartidos por equipos interdisciplinarios de docentes.
De esta manera, las universidades podrán contribuir también con la formación de los contadores públicos del futuro. 


\section{Referencias}

Gómez, A. (2009). Métodos de valuación a valor razonable y su auditoría. CAPIC Review. Vol (7): 35-43.

Gómez, O y R. Álvarez. (2013). Mediciones a valor razonable en la contabilidad financiera. Revista Javeriana. Vol (14): 441-461.

Gómez, O; B. De la Hoz y M. López (2011). Valor razonable como método de medición de la información financiera. Revista Venezolana de Gerencia. Vol (16): 608-621.

González, A.; R. Martínez y T. Requiterena. (2010). Impacto en los estados contables de la medición de cereales y oleaginosos en contextos de incertidumbre. Tesis de Licenciatura. Universidad de la República, Montevideo.

International Accounting Standard Board (IASB). 2010. Marco conceptual para la Información Financiera. Londres, Reino Unido: Fundación del Comité de Normas Internacionales de Contabilidad.

International Accounting Standard Board (IASB). 2011. Normas Internacionales de Información Financiera. Londres, Reino Unido: Fundación del Comité de Normas Internacionales de Contabilidad.

International Federation of Accountants (IFAC). 2011. Normas Internacionales de Auditoría. México, D.F.: Instituto Mexicano de Contadores Públicos, A.C.

Jeppesen, K y D. Liempd (2015). El valor razonable y la conexión perdida entre contabilidad y auditoría. Revista científica general José María Córdoba. Vol (13): 135-171.
Mantilla, S. (2008). Contabilidad y auditoría del valor razonable. Disponible en: https:// www.google.com/webhp?sourceid=chromeinstant \&ion $=1 \&$ espv $=2 \&$ ie $=U T F-$ 8\# $\mathrm{q}=$ mantilla $\% 20$ contabilidad $\% 20 \mathrm{y} \% 20$ auditor\%C3\%ADa\%20del\%20valor\%20 razonable. Consultado el 23 de enero de 2016.

Mantilla, S y S. Yolima (2004). Auditoría de mediciones y revelaciones hechas a valor razonable: Un análisis a raíz de la lectura de ISA 545. Disponible en: http:// cuadernosdecontabilidad.javeriana.edu.co/ vol4_n_19/vol4_19_4.pdf. Consultado el 23 de enero de 2016.

Morala, B.; J. Fernández; Y. Fernández y C. Gutiérrez. (2010). El auditor ante el criterio del valor razonable. Revista Partida Doble. Vol (21): 72-84.

Silva, B. (2011). Valor razonable: un modelo de valoración incorporado en las Normas Internacionales de Información Financiera. Revista Estudios Gerenciales. Vol (27): 97114.

Silva, B y D. Azúa. (2006). Alcances sobre el concepto de valor razonable. CAPIC Review. Vol (4): 61-74.

Suárez, J y P. Lorca. (2007) Las barreras a la expansión del valor razonable. Revista de Contabilidad y Dirección. Vol (4): 147-164.

Tejada, M. (2012). El rol del auditor ante el nuevo estatus del fair value: Tres miradas diferentes del mercado. Disponible en: http://repositorio. udesa.edu.ar/jspui/bitstream/10908/2521/ 1/\%5bP\%5d\%5bW\%5d\%20Tejada\%2c\%20 Mar\%C3\%ADa\%20Yamila.pdf. Consultado el 23 de enero de 2016.

Valle, C. (2009) Hacia el reino de la subjetividad: una crítica al modelo contable del valor razonable. Revista Internacional de Contabilidad y Auditoría. No. 38. 133-154. 


\section{Anexo 1}

Algunas partidas del Estado de Situación Financiera medibles según el valor razonable

\begin{tabular}{|c|c|c|c|c|c|}
\hline \multirow[b]{2}{*}{ Norma } & \multirow[b]{2}{*}{ Partida } & \multicolumn{2}{|c|}{ Valor razonable } & \multicolumn{2}{|c|}{ Tratamiento } \\
\hline & & $\begin{array}{l}\text { Medición } \\
\text { inicial }\end{array}$ & $\begin{array}{l}\text { Medición } \\
\text { porterior }\end{array}$ & $\begin{array}{l}\text { Opcional o } \\
\text { alternativo }\end{array}$ & Obligatorio \\
\hline NIC 9 & $\begin{array}{l}\text { Instrumentos } \\
\text { financieros al valor } \\
\text { razonable con cambios } \\
\text { en resultados }\end{array}$ & $\mathrm{X}$ & $\mathrm{X}$ & & $\mathrm{X}$ \\
\hline NIC 16 & $\begin{array}{l}\text { Propiedad, planta y } \\
\text { equipo }\end{array}$ & & $\mathrm{X}$ & $\mathrm{X}$ & \\
\hline NIC 17 & $\begin{array}{l}\text { Arrendamientos } \\
\text { financieros }\end{array}$ & $\mathrm{X}$ & $\mathrm{X}$ & $\mathrm{X}$ & \\
\hline NIC 20 & $\begin{array}{l}\text { Subvenciones del } \\
\text { Gobierno }\end{array}$ & $\mathrm{X}$ & $\mathrm{X}$ & & $\mathrm{X}$ \\
\hline NIC 38 & Activos intangibles & & $\mathrm{X}$ & $\mathrm{X}$ & \\
\hline NIC 40 & $\begin{array}{l}\text { Propiedades para } \\
\text { inversión }\end{array}$ & & $\mathrm{X}$ & $\mathrm{X}$ & \\
\hline NIC 41 & Activos biológicos & $\mathrm{X}$ & $\mathrm{X}$ & & $\mathrm{X}$ \\
\hline NIF 5 & $\begin{array}{l}\text { Activos no corrientes } \\
\text { mantenidos para la } \\
\text { venta }\end{array}$ & $\mathrm{X}$ & $\mathrm{X}$ & $\mathrm{X}$ & \\
\hline
\end{tabular}




\section{Anexo 2}

Análisis comparativo por variable de las técnicas de ajuste a la tasa de descuento y del valor presente esperado, según el enfoque del ingreso (NIIF 13)

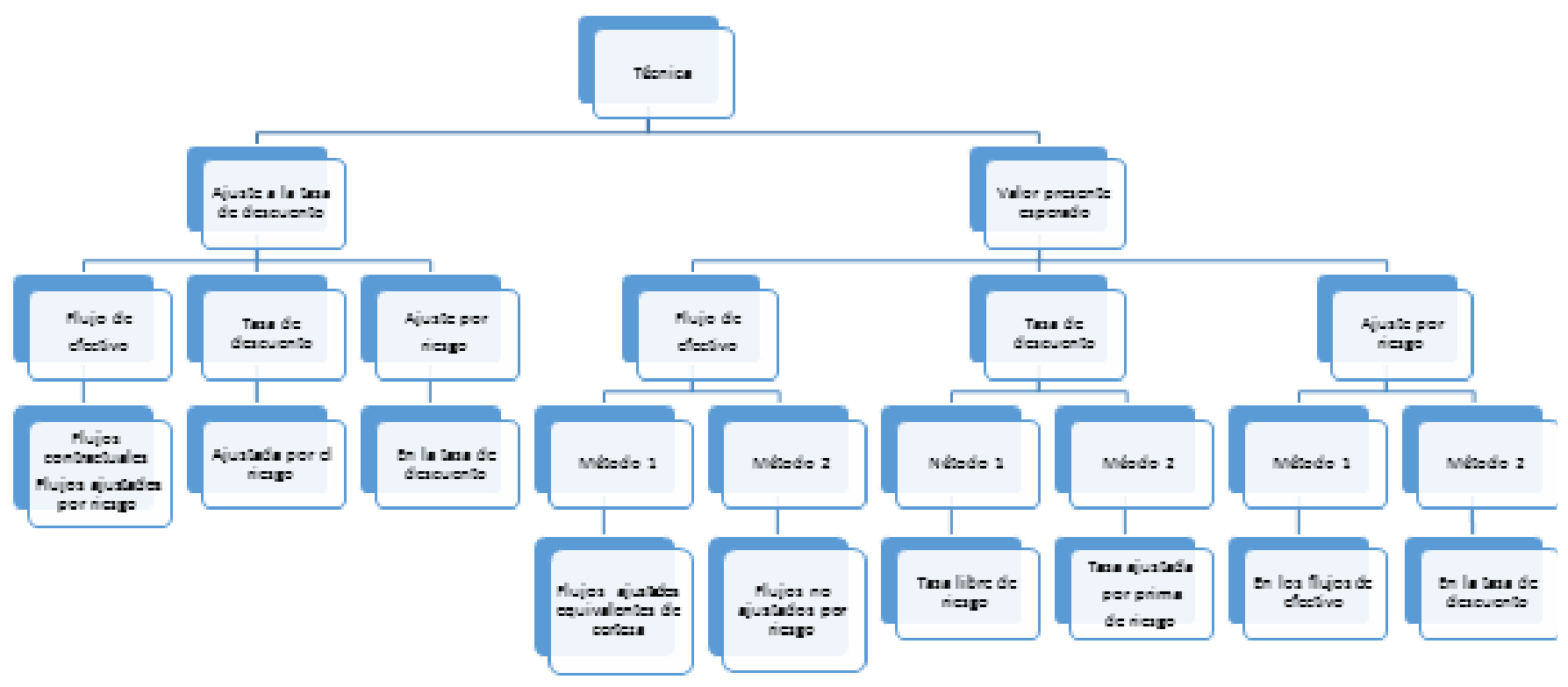




\section{Anexo 3}

\section{Objetivos y procedimientos básicos de auditoría sugeridos para el examen de valores razonables}

\section{Objetivo 1}

Determinar si la metodología y los modelos utilizados por la dirección permiten realizar mediciones adecuadas de los valores razonables de los activos y pasivos

\section{Procedimientos}

Indague con la dirección sobre la metodología, los modelos y los procedimientos específicos establecidos para calcular los valores razonables de los activos y pasivos.

Identifique los mercados principales en que opera la entidad, para efectos de la medición de los valores razonables de los diferentes activos y pasivos. Determine si estos son mercados activos.

Identifique cuál es el mercado el mercado principal y/o el mercado más ventajoso para cada uno de los activos y pasivos que han sido medidos según el valor razonable.

Compruebe que los precios de mercado utilizados para calcular los valores razonables, correspondan a bienes o pasivos idénticos o similares del mercado principal o del más ventajoso.

Compruebe si los precios seleccionados por la empresa para determinar los valores razonables corresponden a los del mercado principal (o el más ventajoso) donde se podría negociar el activo o pasivo respectivo.

\section{Objetivo 2:}

Determinar si los valores razonables por los cuales se presentan los activos y pasivos son consistentes con la

\section{Procedimientos}

Verifique si los cálculos de los valores razonables de activos y pasivos se ajustan a la metodología establecida por la dirección. Compruebe la exactitud de los cálculos aritméticos.

Verifique si los cálculos de los valores razonables de activos y pasivos se ajustan a la metodología establecida por la dirección. Compruebe la exactitud de los cálculos aritméticos.

Evalúe la confiabilidad de las fuentes de datos de las variables y concluya respecto a si estas son representativas de los precios de mercado relevantes

\begin{tabular}{|l|l} 
Hecho por & Referencia \\
\hline & \\
& \\
&
\end{tabular}




\section{Mediciones de valores razonables según el enfoque del ingreso}

\section{Objetivo 1}

Determinar si la metodología y los modelos utilizados por la dirección y las hipótesis en que estos se fundamentan permiten obtener mediciones adecuadas de los valores razonables de los activos y pasivos

\section{Procedimientos}

Indague con la dirección sobre la metodología, los modelos, los supuestos (hipótesis) y los procedimientos específicos establecidos para calcular los valores razonables de los activos y pasivos.

Indague con la dirección sobre las razones que justifican la selección de la metodología y los modelos adoptados para la medición de valores razonables de los diferentes activos y pasivos, así como los supuestos o hipótesis en que se fundamentan las estimaciones.

Identifique otras metodologías alternativas válidas para el cálculo de los valores razonables de los activos y pasivos de la entidad. Señale las razones por las cuales estas no fueron seleccionadas por la dirección.

Compruebe que no se disponga de información sobre precios de mercado (variables observables), de manera que se justifique la aplicación del enfoque del ingreso para el cálculo de valores razonables.

Evalúe la razonabilidad de los supuestos o hipótesis en que la dirección se apoya para determinar los valores razonables, de acuerdo con las condiciones de los mercados en que se venderían los activos o serían transferidos los pasivos.

Evalúe la viabilidad de las hipótesis de la dirección en relación con las condiciones de los respectivos mercados, especialmente en lo referente a:

Niveles esperados de los ingresos

Volumen de producción y ventas

Tasa de crecimiento de los ingresos

Composición de los costos y gastos.

Ritmo de crecimiento de los costos y gastos

Estructura de costos. Costos fijos / costos variables

Márgenes de utilidad

Horizonte temporal de las proyecciones

Valores residuales

Tasas de depreciación y escudos fiscales.

Probabilidades de ocurrencia atribuidas a los diferentes escenarios de las proyecciones.

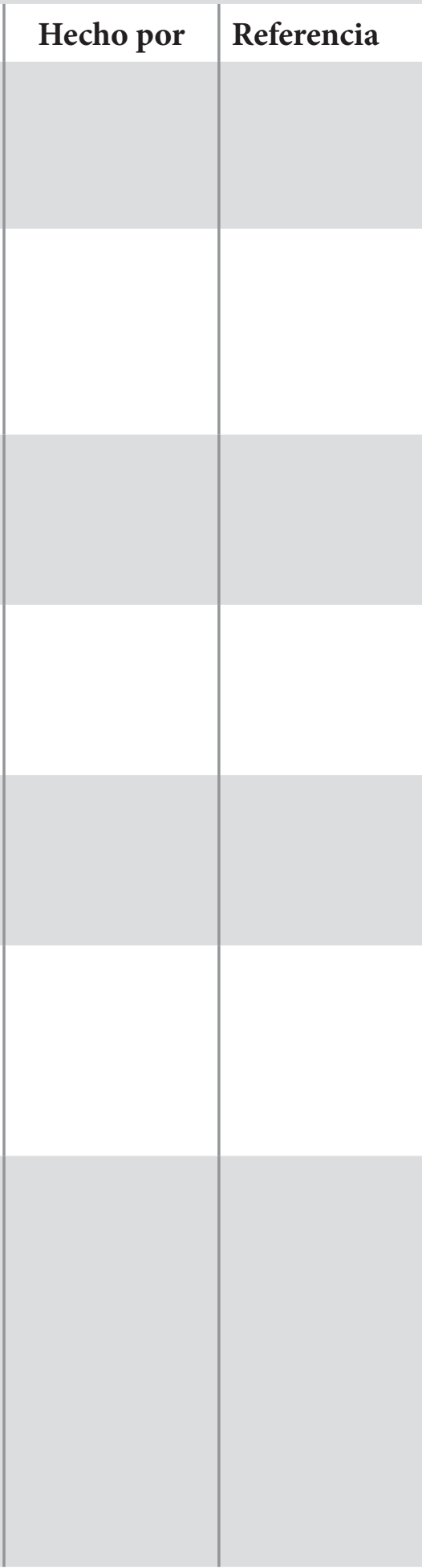


Evalúe si las tasas de utilizadas para descontar a valor presente los flujos de efectivo estimados son razonables y representan adecuadamente el rendimiento exigido sobre los activos y pasivos, considerando el valor cronológico del dinero y el riesgo.

Compruebe que los cálculos de los valores razonables obtenidos son consistentes con la metodología con las hipótesis de la dirección.

IIdentifique las variables críticas o más relevantes del modelo de estimación y realice un análisis de sensibilidad de los resultados obtenidos ante Cambios en esas variables. Concluya acerca del riesgo de estimación.

Realice estimaciones independientes, puntuales o por rangos, de los valores razonables y confronte los resultados con los respectivos saldos en los estados financieros. Discuta los resultados obtenidos con el equipo de auditores y analice las diferencias obtenidas con la dirección.

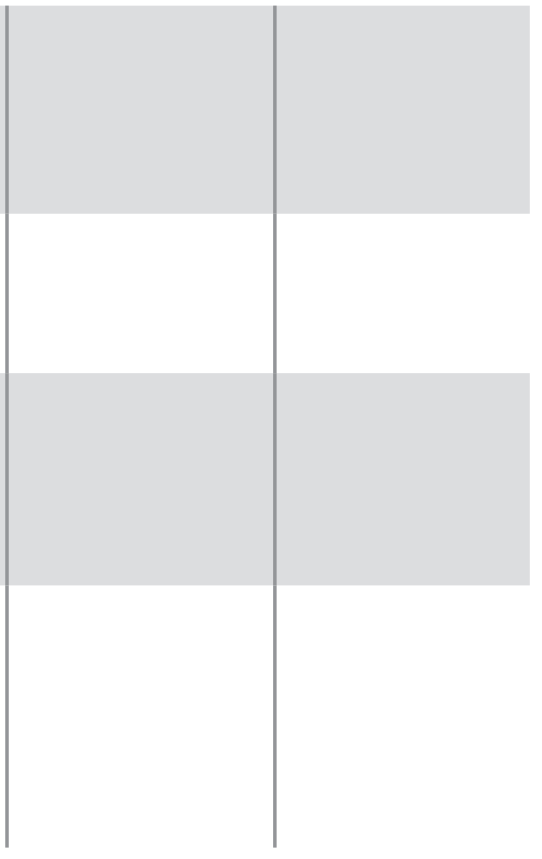


